\title{
Pyogenic granuloma of buccal mucosa mimicking as traumatic fibroma in pregnancy
}

\author{
Rajeev Pandey ${ }^{1}$, Rajat Gupta ${ }^{2}$, Siddharth Rawat ${ }^{3}$
}

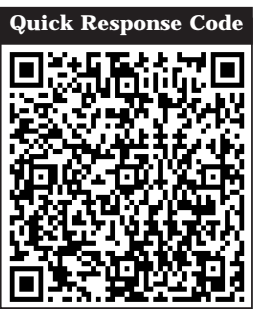

doi: $10.5866 / 2016.8 .10172$

1\&2Senior Lecturer

3Post Graduate Student

Department of Oral and Maxillofacial Surgery

School of Dental Sciences, Sharda University

Greater Noida, UP, India

\section{Article Info:}

Received: J uly 12, 2016

Review Completed: August 11, 2016

Accepted: September 12, 2016

Available Online: December, 2016 (www.nacd.in) (c) NAD, 2016 - All rights reserved

\section{Email for correspondence:}

rajeevpandeyleo@gmail.com

\begin{abstract}
:
Pyogenic Granuloma (PG) is one of the commonly occurring non neoplastic lesion in oral cavity. The most common site for occurrence is gingiva and occurrence at extragingival sites is rare. PG occurring extragingivally presents a diagnostic dilemma. The etiology for PG is multifactorial which includes low grade chronic irritation, hormonal imbalance, trauma and medications such as cyclosporine. PG has predilection to occur during pregnancy over the gingival region. This is due to the hormonal changes which reduce the healing capacity of keratinizing mucosa especially gingival. Therefore occurrence of extragingival PG in pregnancy without any gingival PG can lead to confusion in diagnosis. A case of PG mimicking a traumatic fibroma of extragingival site, buccal mucosa, in a 25 year old pregnant female is presented in this paper. The lesion was provisionally diagnosed as traumatic fibroma and was surgically removed using electro cautery. Histopathologically the lesion was reported as pyogenic granuloma. Post surgically the site was healed completely and during one month follow up there was no recurrence.
\end{abstract}

Key words: Pyogenic granuloma, buccal mucosa, electrocautery

\section{INTRODUCTION}

Soft tissue lesions of oral cavity are a common occurring phenomenon. They represent a diverse range of pathologic processes including reactive lesions, variation of normal anatomic structure, inflammatory lesions, cysts, neoplasm and developmental anomalies. Sometimes these lesions may have overlapping clinical features leading to diagnostic challenge. Therefore a sound knowledge about these lesions is essential for ideal treatment and to prevent recurrence. Reactivelesions are most routinely found soft tissuelesions of oral cavity. They are commonly found on tissues which are injured. These lesions are commonly represented by: Pyogenic granuloma, peripheral ossifying fibroma, traumatic fibroma and peripheral giant cell grauloma. PG is the most commonly occurring reactivelesion. ${ }^{1}$ It is a nodular growth of granulation tissue which can arise from intraoral mucosa or sometimes from skin surface. It is basically a lesion of gingiva (two third of the cases) and extragingival occurrence is a rarity. There is a strong association 
between pregnancy and PG. It is seen in about $5 \%$ of pregnancies. In pregnancy the PG may start developing during the first trimester with increased incidence as pregnancy progress. ${ }^{2}$

This is due to the fact that hormonal fluctuations during pregnancy increase the influence of local irritational factors leading to patient more prone for PG. Occurrence of extragingival PG in a pregnant patient without any gingival $P G$ is a very uniquesituation. The presentation becomes atypical and confusing and the lesion may mimic other benign soft tissue growth and sometimes may resemble even malignancy. ${ }^{3} \mathrm{~A}$ case of extragingival PG of buccal mucosa in a 25 year old female with second trimester pregnancy mimicking like traumatic fibroma is presented in this paper. The purpose of this case report is to highlight the importance of biopsy for confirm diagnosis and pre operative planning needed to prevent intraoperative and postoperative complications.

\section{CASE REPORT:}

A 25 year old female reported to us with chief complaint of a growth in the right buccal mucosa since 4 months. The growth was initially small in size and had enlarged progressively to a size large enough to interfere with her normal occlusion. The patient admitted that she has habitual cheek biting habit of right side. She also reported occasional bleeding from the lesion but no pain. The patient was five month pregnant and it was her first pregnancy.

\section{Clinical Presentation:}

On clinical examination there was an irregularly shaped, pedunculated growth in the right buccal mucosa $2 \mathrm{~cm}$ above 46,47 teeth at the level of occlusal plane, measuring about $2.5 \mathrm{~cm} \times 2 \mathrm{~cm}$ (Figure 1). The surface of the lesion was lobulated and was predominantly reddish whitein colour with focal areas of ulcerations. On palpation, the lesion was found to be non tender, firm in consistency, with no signs of any discharge. There were no visible or palpable pulsations observed. There was no other lesion in oral cavity including gingival. The overall oral hygiene was good and mild gingivitis was present. Extra oral examination was not contributory. Based on the history and clinical presentation, we arrived at a provisional diagnosis of traumatic fibroma of the right buccal mucosa because of history of cheeking biting habit and presence of lesion at the occlusal level. The differential diagnosis included capillary hemangioma and pyogenic granuloma. Routine hematological investigations were performed and found to be in the normal range. An excisional biopsy was planned. As the patient was second trimester pregnant, this is relatively safer to perform routine dental procedures under local anesthesia. The patient was planned to be operated in a left lateral decubitus position to avoid pressure over inferior vena cava preventing hypotension. As thelesion was reddish and appered quite vascular we planned to perform the excisional biopsy using el ectocautery (Bonart Electron Art-E 1 Electrosurgery Unit) to minimize bleeding intraoperatively and postoperatively (We found no contra indication for use of electrosurgery in pregnancy in literature review).

\section{Technique:}

Under aseptic conditions the patient was draped and electrosurgery unit was connected as per operator manual. Patient was made to do betadine gargle two times. An elliptical incision was marked around the lesion using marking ink. Local infiltration was injected around the lesion. Before contacting the electrode with tissue, suitable power intensity was chosen. Before starting el ectrosection the lesion was moistened with help of normal saline to prevent tissue charring. The lesion was grasped using toothed forcep and retracted. Incision was placed around the lesion as per the marking and lesion was removed from the mucosa and underlying tissues. There was hardly any bleeding seen. Primary closure was done using 3-0 silk using non cutting needle. Routine post operative instructions were given and patient was discharged. Cap amoxicillin 500mg TDS and Tab. Paracetamol $500 \mathrm{mg}$ TDS was prescribed for 5 days. Patient was also advised betadine mouth gargles from next day onwards. She was recalled after 7 days for suture removal. Suture removal was done after 7 days. The patient was recalled after 1 month and no recurrence was seen (Figure 2). 


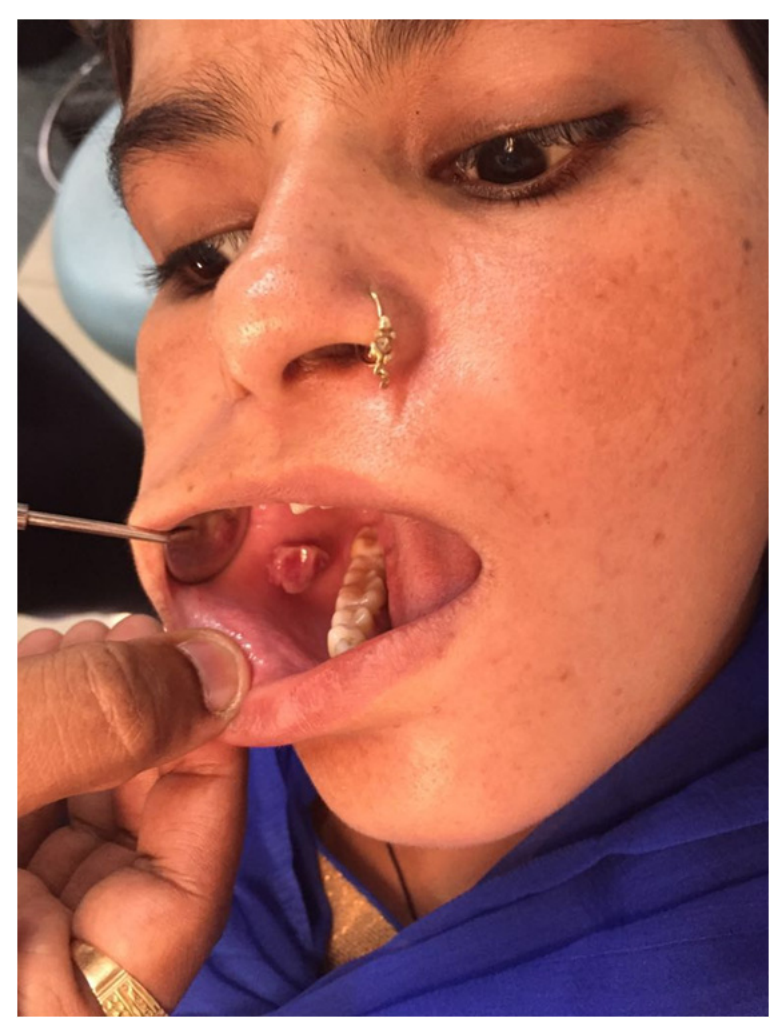

Figure 1: Clinical picture showing an irregularly shaped, pedunculated growth in the right buccal mucosa $2 \mathrm{~cm}$ above 46 , 47 teeth at the level of occlusal plane, measuring about $2.5 \mathrm{~cm} \mathrm{X}$ $2 \mathrm{~cm}$

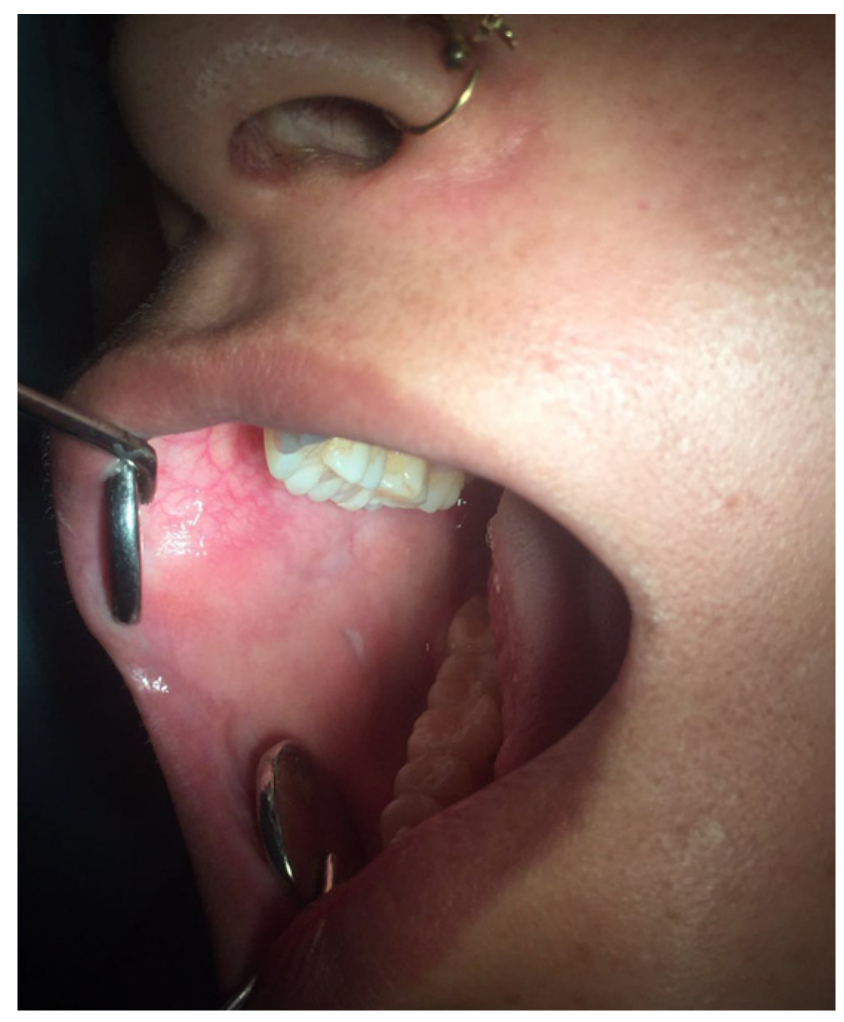

Figure 2: Post operative picture showing complete healing and no recurrence was seen after one month.

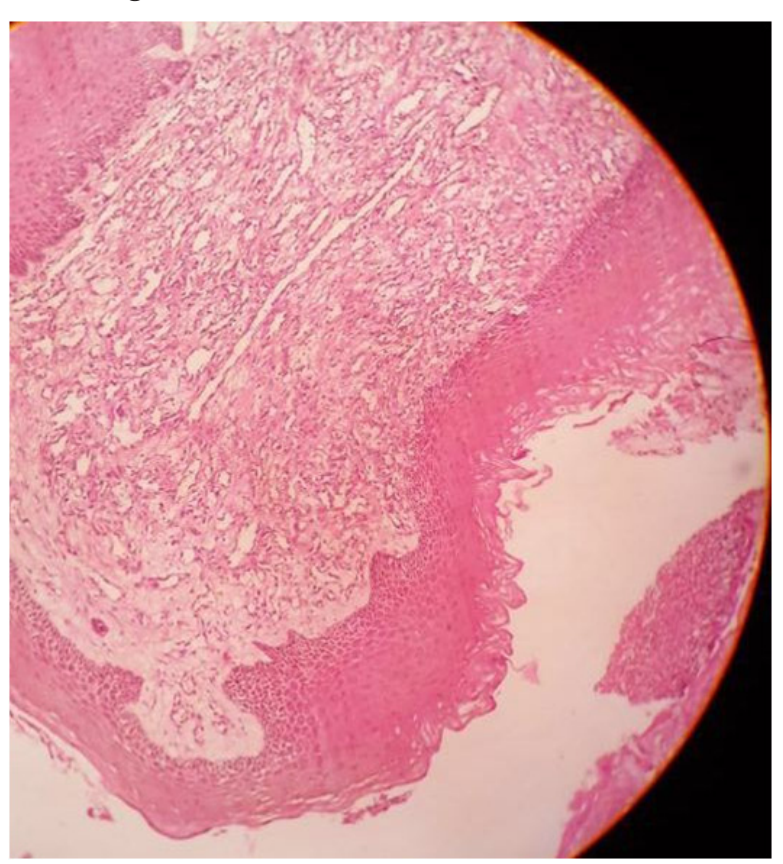

Figure 3: $\mathrm{H}$ and $\mathrm{E}$ stained section shows hyperplastic parakeratinized stratified squamous epithelium with underlying connective tissue stroma showing numerous endothelium lined blood vessels along with extravasated RBCs and dense inflammatory cell infiltrate predominantly composed of lymphocytes. It also shows collagen bundles with plump fibroblasts interspersed suggesting pyogenic granuloma. 


\section{Histopathology:}

$\mathrm{H}$ and $\mathrm{E}$ stained section showed hyperplastic parakeratinized stratified squamous epithelium. The underlying connective tissue stroma showed numerous endothelium lined blood vessels with extravasated RBCs. Dense inflammatory infiltrate predominantly lymphocytes were seen. It also showed collagen bundles with plump fibroblasts interspersed. These findings were suggestive of pyogenic granuloma (Figure 3).

\section{DISCUSSION:}

Extragingival occurrence of PG is a rarity and less than one third of the cases occur extragingivally. ${ }^{4}$ For extragingival occurrence, tongue (4\%) and lower lip (3\%) are most commonly involved. Occurrence on buccal mucosa is rare. The occurrence of PG is higher in pregnancy. It is reported that in pregnancy $5 \%$ of females show PG. This is generally applicable for gingival PG. Occurrence of extragingival PG in pregnancy has also been reported. ${ }^{5}$ In pregnancy presence of local irritants along with hormonal factors increases the probability of development of PG, mainly in the gingiva. There is an increased amount of estrogen and progesterone which increases the vascular response potential. For devel opment of extragingival PG in pregnancy trauma is the main etiology. This was seen in the above case also, as the patient had habit of cheek biting which has probably lead to the formation of $P G$.

Clinically there is no differentiating feature of PG from other intra oral soft tissue lesions. They have a complex clinical presentation ranging from highly vascular presentation to a more fibrotic one. In pregnancy they appear more vascular with tendency of spontaneous bleeding. ${ }^{3}$ The clinical presentation in our case was of a vascular lesion with history of spontaneous bleeding, but there was no other soft tissue lesion in the oral cavity especially on gingiva. Only a single mass was present over buccal mucosa at the level of occlusion surface. Therefore a provisional diagnosis of traumatic fibroma was made as the patient had history of traumatic bite. There was clinical diagnostic dilemma because of site of occurrence and clinical appearance. The lesion was causing difficulty in mastication so it was planned for surgical excision. Due to the reddish color of the lesion representing increased vascularity and anticipating bleeding while removal of the lesion therefore we planned to remove the lesion using electrosurgical unit to minimize bleeding. The lesion was removed successful without bleeding and post operatively also the healing was uneventful. During one month follow up there was no recurrence.

\section{CONCLUSION:}

Biopsy is the confirmatory diagnostic aid for intra oral soft tissue lesions. Pre operative planning is important to minimize bleeding if such a event is anticipated. U se of modern surgical aids rather than surgical blade helps in better surgical procedureand patient comfort if soft tissue lesions are removed this which are prone for bleeding.

\section{REFERENCES}

1. Saravana $\mathrm{GH}$. Oral pyogenic granuloma: a review of 137 cases. Br J Oral Maxillofac Surg 2009; 47:318-9.

2. Silverstein $\mathrm{LH}$, Burton $\mathrm{CH} J \mathrm{r}$, Singh BB. Oral pyogenic granuloma in pregnancy. Int J Gynaecol Obstet 1995; 49:331-2.

3. Krishnapillai R, Punnoose K, Angadi PV, Koneru A. Oral pyogenic granuloma-a review of 215 cases in a South Indian Teaching Hospital, Karnataka, over a period of 20 years. Oral Maxillofac Surg 2012; 16:305-9.

4. Pandey R, Gupta R, Bhagat N, Verma A. Pyogenic granuloma as a posterior maxillary swelling in edentulous region: a rare case report. Int J Contemporary Med Res 2016; 3:1816-7.

5. Cardoso J A, Spanemberg J C, Cherubini K, de Figueiredo MAZ, Salum FG. Oral granuloma gravidarum: a retrospective study of 41 cases in Southern. Brazil J Appl Oral Sci 2013; 21:215-8.

\section{Gain quick access to our journal online View our journal at www.nacd.in}

